\title{
LE TOMBEAU D'AMPHION ET DE ZÉTHOS ET LES FRUITS DE DIONYSOS
}

\section{Maria Rocchi}

Pausanias raconte que le tombeau d'Amphion et de Zéthos à Thèbes de Béotie était un "tumulus pas grand" et il s'étend sur le récit de ce que, chaque année, Thébains et Tithoréens auraient fait autour de ce tombeau pour appeler la fertilité sur leurs champs respectifs. ${ }^{1}$

Toujours selon Pausanias, quand le soleil se trouvait dans la constellation du Taureau, les habitants de Tithorée en Phocide projetaient de dérober de la terre au tumulus thébain d'Amphion et de Zéthos pour la porter sur la tombe d'Antiope dans leur propre cité, et ceci afin d'obtenir une bonne récolte au détriment des Thébains qui, de leur côté, devaient surveiller leur tumulus.

Le comportement des uns et des autres se baserait sur un oracle de Bakis dont Pausanias nous rapporte le texte et il ajoute qu'Antiope, la mère des deux héros, était ensevelie à Tithorée avec Phokos, son dernier époux. ${ }^{2}$

De nombreux savants ont interprété le récit de Pausanias, mais leurs hypothèses ne nous semblent pas tout à fait convaincantes.

J.G. Frazer, commentant le texte de Pausanias, avance l'hypothèse que la période indiquée pour la démarche des Tithoréens correspondait à l'époque des semailles et il rappelle à ce propos que, dans la Rome antique, les Lois des Douze Tables interdissaient, entre autre, de dérober par des pratiques magiques la récolte du champ d'autrui. ${ }^{3}$

F. Vian voit, dans ce qu'il définit comme un "curieux rite agraire", une confirmation des prérogatives qui situeraient les jumeaux Amphion et Zéthos, bergers et bâtisseurs de murailles, dans la "troisième fonction" dumézilienne de la civilisation indo-européenne. ${ }^{4}$

W. Burkert pense, lui, à une forme résiduelle d'un rite de passage transposée sur le plan magique et il la met en relation avec un autre rite nocturne, celui célébré par les Hipparques thébains près de la tombe de Dirké, au moment de la passation des pouvoirs. 5

A. Schachter, de son côté, pense que Pausanias ne se réfère pas à un rite effectivement célébré de son temps, mais qu'il aurait inventé cette histoire à partir du texte de Bakis. ${ }^{6}$ 
Il nous semble donc nécessaire d'analyser de près les témoignages des Anciens afin de mieux comprendre la situation cultuelle, objet de notre étude.

L'oracle de Bakis dit textuellement: "mais quand un homme de Tithorée offre des libations pour Amphion et Zéthos, des prières et des offrandes propitiatoires, dans la période où le Taureau est ensorcelé par la force du glorieux soleil, alors toi, prends bien garde qu'un grand mal ne s'abatte sur la cité car, en effet, les fruits s'abîmeront ici si les Tithoréens partagent la terre et s'ils la transportent sur le tombeau de Phokos".7

On appelait communément, "bakides" les thaumaturges et les devins possédés par les dieux ${ }^{8}$ et parmi eux la tradition et Pausanias en indiquaient un de Béotie. ${ }^{9}$

On peut aussi attribuer à un devin béotien l'oracle qui nous intéresse étant donné qu'il est adressé à un homme de Thèbes, qu'il concerne le tombeau des Dioskouroi de cette cité et qu'il est prononcé dans lé but de ne pas laisser les Thébains perdre les fruits de leur terre.

Selon Bakis il s'agit d'un danger menaçant dont, à cette époque précise, les Thébains doivent se garder: un homme de Tithorée donne des offrandes sur le tombeau d'Amphion et de Zéthos et les Tithoréens partagent $(\delta \alpha \sigma \sigma \alpha \mu \varepsilon v \omega v)^{10}$ la terre du tumulus pour emporter leur part sur le tombeau de Phokos.

Dans sa description de Thèbes, Pausanias cite le texte de l'oracle de Bakis et l'enrichit de détails.

Dans le récit de Pausanias, il convient avant tout de noter que le danger annoncé par l'oracle n'avait pas un caractère exceptionnel mais qu'il se répétait chaque année, chaque fois que le soleil dans sa course à travers la Zodiaque qui détermine la succession des mois, revenait dans la constellation du Taureau. L'action des Tithoréens devait consister à dérober en cachette, contre la volonté des Thébains ( répandre autour ( $\pi \varepsilon \rho\llcorner\alpha ́ \phi w \sigma \iota)$ de la tombe d'Antiope et de Phokos, et rendre ainsi plus féconde leur récolte. Tout ceci au détriment des Thébains qui, de leur côté, auraient dû surveiller le tumulụs, suivant "à la lettre" l'ordre de Bakis."

Il ne ressort, ni du texte de Bakis ni de celui de Pausanias, que les habitants des deux cités se soient jamais réellement affrontés autour du tumulus. 
Il apparaît invraisemblable que pour célébrer un rite l'on ait pu chaque année, voler de la terre à un tumulus "pas grand" et courir le :risque d'irriter les héros et même celui de les faire revenir puisque, en enlevent de la terre, on amincit le diaphragme qui separe les morts du monde des vivants. ${ }^{12}$

A notre avis, l'hypothèse la plus probable est que les Tithoréens avaient l'intention de partager le tumulus d'Amphion et de Zéthos avec les Thébains et, une fois parvenus à leurs fins, d'en répandre, une fois pour toutes, la terre en cercle ${ }^{13}$ autour de la tombe d'Antiope et de Phokos, réunissant ainsi les fils avec la mère, ce qui leur aurait valu en échange une fertilité accrue de leurs champs.

A notre avis, en outre, l'oracle de Bakis, qui s'adresse aux Thébains, n'aurait servi qu'à repousser avec autorité les intentions des Tithoréens. En montant simplement bonne garde autour du tumulus, les Thébains auraient conjuré le danger d'être dérobés, d'être frappés par la colère d'Amphion et de Zéthos et de voir dépérir les fruits de leur terre. ${ }^{14}$

Il arrive souvent dans le monde grec que des héros soient ensevelis dans plusieurs endroits; que des cités se disputent les tombes de morts illustres ${ }^{15}$ ou que l'une d'elles, se conformant à la volonté des dieux exprimée par un oracle, réussisse même à transférer les os d'un héros sur son propre territoire. ${ }^{16}$

Il se pourrait que dans notre cas il s'agisse aussi d'une controverse pour la possession d'une tombe. On attribuait, en effet, plusieurs demeures aux morts Amphion et Zéthos. On disait qu'ils étaient ensevelis à Thèbes près des portes Proitides, hors des murailles ${ }^{17}$ qu'ils avaient eux-mêmes bâties; ou encore que, nés de Zeus et d'Antiope, ils étaient un couple de jumeaux dioscuriques ${ }^{18}$, c'est à dire en mesure, comme Kastor et Polydeukes, d'aller et de venir entre leur demeure souterraine et celle qu ils auraient eue parmi les étoiles ${ }^{19}$; ou, enfin, qu'ils avaient une tombe à Tithoraia. ${ }^{20}$ Cette dernière information, même si elle n'était que construite par Stephanos de Byzance à partir du texte de Pausanias, confirmerait notre hypothèse sur l'origine du récit du Périégète, à savoir un conflit entre deux cités pour la possession d'une tombe.

Il nous reste à voir pourquoi l'état d'alerte se vérifiait autour du tombeau des héros à une certaine période de l'année et quels étaient les fruits menacés. 
La période pendant laquelle le soleil se trouve de nos jours dans la constellation du Taureau s'étend du 21 avril au 21 mai.21

Deux groupes d'étoiles forment dans le ciel la figure ouranienne du Taureau: les Hyades représentent le bucrane et les Pléiades la partie terminale, étant situées près de la queue ou à mi-dos là ou le corps de l'animal, visible dans sa seule partie antérieure, est coupé. 22

Les deux groupes d'étoiles étaient mis en relation avec Dionysos. Les Hyades auraient été les nourrices du dieu 23 ; et elles auraient donné la vigne aux hommes. ${ }^{24}$ Les Pléiades, elles aussi nourisses du dieu, apparaissaient dans le ciel comme un "botrys", une grappe de raisin..$^{25}$

Pour comprendre à quels fruits se référait l'oracle, il est nécessaire de se reporter aux travaux agricoles qui occupaient les Thébains quand le soleil se trouvait dans la constellation du Taureau.

J.G. Frazer (loc. cit.) pensait qu'il s'agissait des semailles de printemps car on disait, en effet, que le paysan devait labourer pendant cette période. ${ }^{26}$

Il semble toute fois qu'il faille écarter les "fruits de Demeter" qu'ils vont être moissonnés quand le soleil se trouve dans les Didymoi.

Par contre, c'est aux vignes qu'il faut consacrer un grand soin en avril-mai à cause de la floraison et pour aider la formation des fruits. ${ }^{27}$

Hésiode dans "Les Travaux" donne à ce propos un précepte qu'il introduit par une formule oraculaire (cfr. West ad 571) à la manière de Bakis: "Mais, quand le porte-maison monte de la terre à l'escalade des arbres fuyant devant les Pléiades, ce n'est plus le temps de piocher les vignes. Aiguisez les faucilles et éveillez vos serviteurs...au temps de la moisson, quand le soleil sèche la peau. C'est l'heure de faire vite, de ramasser votre récolte." (trad. Mazon vv. 571-575; cfr. 383 ss.).

La scholie à ces vers fournit les motifs techniques de l'exhortation d'Hésiode: après le "lever matinal" ou epitolé des Pleiades quand le soleil entre dans la constellation des. Didymoi, il ne faut plus piocher la vigne; la terre, en effet, est desséchée et les racines, exposées au soleil, seraient abîmées par la chaleur de l'astre et il ne faut plus tailler la plante mais la laisser nourrir en paix ses fruits. ${ }^{28}$

L'epitolé, c'est à dire l'apparition des Pleiades dans le ciel quelques instants avant l'aube, a lieu au mois de mai $^{29}$ quand le soleil se trouve dans la constellation du Taureau. ${ }^{30}$ 
L'exhortation d'Hésiode de ne pas travailler la vigne est donc motivée, non seulement par le fait que, à cette époque, c'est la fauche du blé qui doit occuper les ouvriers aux champs, mais aussi par les conditions particulières des vignes.

C'est à peu près au "lever matinal" des Pleiades qu'ils arrivent dans les vignobles les jours de la floraison. ${ }^{31}$

On apprend à ce propos que les vignerons chargés d'émonder les pampres, s'abstenaient de ce travail au mois de mai pendant la floraison. ${ }^{32} \mathrm{On}$-empêchait même d'entrer dans les vignobles pour éviter d'abîmer les plantes. ${ }^{33}$ On prenait les mêmes précautions pour le piochage des vignes, pour ne pas risquer d'abîmer les fruits.

Par contre, à qui n'avait pas coutume de piocher souvent la vigne à chaque période de l'année, ${ }^{34}$ on recommandait de le faire au moins trois fois par an dont l'une, précisément, dans la période qui nous intéresse. Le remuage de la terre en avril-mai ${ }^{35}$ avant lai floraison ou le "lever matinal" des Pléiades, ${ }^{36}$ avait pour but d'assurer une nourriture supplémentaire à la plante pour l'aider dans la formation des fruits.

On peut donc déduire, de tout ce qui précède, qu'aux environs de l'apparition des Pléiades, lorsque le soleil se trouvait dans la constellation du Taureau, on arrêtait de travailler les vignes à cause de la floraison et que le passage du soleil de la constellation du Taureau à celle des Gémeaux coïncidait avec la phase critique liée à la formation des nouveaux karpoi.

La relation qui existait entre Antiope et ses fils et Dionysos - que les Thébains appelaient "ampelos" c'est-à-dire "vigne" 37 - peut expliquer pourquoi ces personnages mythiques étaient précisément mis en cause dans cette période critique pour la viticulture.

C'est Pausanias ${ }^{38}$ qui fait de Dionysos l'arbitre de leur vicissitude. Antiope, dit-il, aurait été ensevelie à Tithorée parce que c'est là qu'elle serait arrivée après que Dionysos l'aurait frappée de folie et fait errer à travers l'Hellade pour la punir de la peine excessive infligée à Dirké qui, à Thèbes, l'aurait eu en grand honneur parmi tous les dieux. Antiope, accueillie et guérie par Phokos, l'aurait épousé et aurait partagé sa tombe une fois morte.

Il convient de résumer ici les traits essentiels du mythe auquel se réfère Pausanias pour comprendre le rôle de Dionysos. 
On disait qu'Antiope, mère d'Amphion et de Zéthos, était fille d'Asopos ou de Nykteus et qu'elle avait comme ancètre Alkyone une des Pléiades ${ }^{39}$ que nous avons vu former le corps du Taureau ouranien.

Zeus l'aurait séduite sous le traits d'un satyre 40 ou d'un taureau, ${ }^{41}$ c'est-à-dire sous une apparence dionysiaque

Antiope donna le jour à deux jumeaux à Eleuthère, dans une grotte qu'on indiquait près du temple de Dionysos au pied du Citheron, la montagne où les Thébains célébraient les rites dionysiaques. ${ }^{42}$

Abandonnés sur le Citheron par Antiope après leur naissance, les jumeaux auraient été élevés par des bergers et ils n'auraient revu leur mère qu'à l'occasion des faits dont parle Pausanias.

Antiope, prisonnière du roi de Thèbes, Lykos et de Dirké, serait parvenue à s'echapper miraculeusement et à rejoindre le Citheron ${ }^{43}$ où elle aurait demandé aide à ses fils. Ceux-ci auraient fait périr Dirké en l'attachant aux cornes d'un taureau, c'est-à-dire de la même façon dont Dirké aurait eu l'intention de tuer elle-même Antiope, ${ }^{44}$ ou de la faire tuer par Amphion et Zéthos, bouviers à son service. ${ }^{45}$

Ce qui nous intéresse plus particulièrement, ce sont les conséquences que cette histoire légendaire a pu avoir pour les Thébains et les Tithoréens.

La mort de Dirké et de son époux Lykos, roi de Thèbes, tué luiaussi par Amphion et Zéthos ou depouillé de son royaume, permit aux fils d'Antiope de devenir rois à leur tour, d'entrer enfin dans la cité et d'avoir l'honneur de ce tombeau.

Un fragment de l'Antiope d'Euripide souligne l'incertitude qui suivit la mort de Dirké: à ce moment-là, Amphion ignore encore s'il va être puni pour l'avoir tuée ou si, avec l'aide de Zeus, son père, il va réussir à vaincre Lykos et à s'emparer du royaume avec son frère. ${ }^{46}$ Hermès intervient alors et explique comment l'ordre fixé à l'avance par Zeus doit être exécuté a Thèbes: Amphion et Zéthos deviendront rois de la cité et seront honorés comme Dioscures ${ }^{47}$; le cadavre de Dirké doit être jeté dans une source qúi prendra son nom. ${ }^{48}$

Après sa mort, Dirké continue donc à jouer un rôle pour les Thébains: c'est dans sa source que naît l'un des deux fleuves qui entourent la cité 49 et où Zeus aurait trempé Dionysos pour le sauver des flammes qui consumaient Sémélé, sa mère. ${ }^{50}$ 
Quant au destin d'Antiope, lié à la mort de Dirké, il intéresse surtout les Tithoréens. Contrainte par Dionysos à abandonner Thèbes, elle épouse Phokos et en partage la tombe à Tithorée. Cette vicissitude d'Antiope quittant la métropole des Bacchantes pour le Parnasse, autre lieu dionysiaque, ${ }^{51}$ pourrait témoigner du rôle joué par la cité de Thèbes dans la diffusion du culte du dieu..$^{52}$ Mais du fait qu'Antiope, une fois guérie de sa folie momentanée, s'établit définitivement auprès du héros qui a donné son nom à la Phocide, ${ }^{53}$ il nous semble que son destin est plutôt semblable à celui des Proitides. ${ }^{54}$

A la fin de cette analyse, on peut donc penser que le danger qui menaçait les Thébains dans la période avril-mai, qu'ils appelaient "Thyios", 55 était le suivant: si les Tithoréens, au nom de cette Antiope qui était arrivée chez eux frappée de folie "thyia", 56 étaient parvenus à s'approprier du tumulus d'Amphion et de Zéthos, l'ordre voulu pour Thèbes par les dieux aurait été renversé. Pour conjurer ce danger, les Thébains, obéissant à l'oracle, montaient bonne guarde chaque année pour empêcher que l'on outrageât leurs Dioscures just'avant que le soleil entre dans le signe zodiacal dans lequel on les reconnaissaient dans le ciel${ }^{57}$ et que Dionysos ne se venge sur les fruits de leur terre, dans son courroux contre Antiope qu'il avait bannie de Thèbes.

\section{Notes}

Je remercie le Professeur Pierre Lévêque pour l'intérêt qu'il a bien voulu manifester à mon étude, ainsi que pour les précieux conseils et suggestions qu'il m'a aimablement donnés.

1 Paus. 9, 17, 3-7; cf. pour les fouilles archéologiques sur le "tumulus d'Amphion" Th. G. Spyropoulos, Ampheion, Sparte 1981.

2 Pausanias mentionne de nouveau le tombeau $(10,32,8-11)$ à propos de Tithorée ville phocidienne dejà en déclin de son temps, située au sommet du Mont Parnasse dont elle tirait son nom (cf. Hdt. 8, 32).

3 J. G. Frazer, Pausanias's Description of Greece, V, London 1898, p. 57 s.; H. Hitzig Bluemner, Pausaniae Graeciae Descriptio III, Lipsiae 1907, p. 438.

4 F. Vian, Les Origines de Thèbes, Paris 1963, p. 135.

5 W. Burkert, Homo necans, Berlin-New York 1972, p. 210; id. Griechische Religion der archaischen und klassischen Epoche, Stuttgart 1977, p. 325.

6 A. Schachter, Cults of Boiotia, BICS Supplement XXXVIII, 1981, p. 29.

7 Paus. 9, 17, 5 .

8 Cf. Pla. Thg. 124 D; Arist. Pr. 30, 1 (954a); Plu. De Pyth. or. 10.

9 Paus. 10, 12, 11; Theop. F. 78 (Grenfell-Hunt).

10 Cf. par exemple Zeus et les dieux aui se partagent la terre (Pi. O. 7, 55 et Sch. adloc.); St. Bvz. s.v. $\delta \alpha \tau \varepsilon_{\text {É }} \alpha_{\iota}$ "divido, partior"; Liddell-Scott-Jones, s.v. "divide among themselves". 


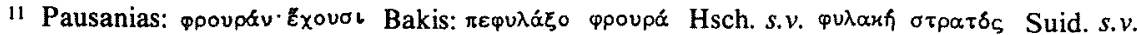

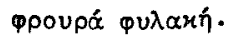

12 A. Schnauter, Frühgriechische Totenglaube. Untersuchungen zur Totenglaube des mykenischen und homerischen Zeit, New York 1970, p. $18 \mathrm{~s}$.

$13 \mathrm{Cf}$. à propos du cercle qu'ils décriraient autour de leurs morts S. Eitrem, Opferritus u. Voropfer der Griechen und der Römer, Kristiania 1915, p. 9-12; A. Schnaufer, op. cit., 19. Il faut remarquer à ce propos, que diverses lectures nous sont proposées par les editeurs du texte de Pausanias: Hitzig-Bluemner conjecture avec Frazer $\pi \varepsilon \rho \iota \tau \iota \theta \tilde{\omega} \sigma \iota \nu$ Spiro lit dans les codices $\pi \varepsilon \rho\lrcorner \alpha ́ \alpha \omega \sigma \iota$; Rocha-Pereira prefere indiquer une lacune.

14 On lit dans les malédictions funéraires que la terre ne doit plus donner de fruits aux violateurs de tombeaux cf. A. Parrot, Malédiction et violation des tombes, Paris 1939, p. 141-143; 151; L. Robert, Malédictions funéraires grecques, CRAI 1978, p. 262 note 117; 270 note $5 ; 283$.

is Cf. F. Pfister, Der Reliquienkult im Altertum, Giessen 1909, p. 193-211; 218 ss; et à ce propos aussi C. Bérard, Récuperer la mort du prince; héroisation et formation de la cité, dans $L a$ mort. les morts, dans les sociétés anciennes, G. Gnoli et J.P. Vernant, Cambridge-Paris 1982, p. 98; F. Hartog, La mort de l'autre, ibid. $143 \mathrm{~s}$.

16 Cf. les cas de Hektor (Lyc. 1204 ss. Sch. ad loc. 1208; Aristodem F. 7 (FGrH); Paus. 9, 18, 5); Rhesos (Polyaen. 6, 53): Hesiodos (Arist. F. 565 Rose) Arkas (Paus. 8, 9, 3-4; 36, 8) Aristomenes (Paus. 4, 32, 3). La confrontation est dejà suggérée par Weniger, Roscher s. $v$. Phokos 2412.

17 Paus. 9, 16, 6; Sch. E. Ph. 145; ou Borrhaiai "du Nord" A. Th. 527 s.

18 Cf. pour Amphion et Zéthos "Dịoscures"; Pherecyd. F. 124 (FGrH); Pi. P. 9, 38; Sch. ad loc.; E. HF. 29 s.; Ph. 606; Antiop. F. 48 (Kambitsis) 98 s.; Hsch. s.v. $\Delta \iota 6 \sigma$ หovoo ; Sch. Hom. Od. 19, 518; A. Schachter, Cults of Boiotia, loc. cit.

19 Hyg. Astr. 2, 22; Ov. Fast. 5, 719 s.; cf. G. Piccaluga, I Dioskouroi: la morte come libera scelta, Il senso del culto dei Dioscuri in Italia, Atti del Convegno di Taranto 1979, Supplemento: Produttività Ionica X, $43 \mathrm{~s}$.

20 St. Byz. s.v. T $\iota$ $\theta 0 \rho \alpha$ l $\alpha$

21 Cf. Eudox F. 245; 258a (Lasserre); 22 avril-21 mai; Gem. Calend. p. 107 (Aujac): 23 avril-24 mai: Ov. Fast. 4, 713-720; 20 avril-19 mai; Colum. Rust. 11, 2, 36; 43.

22 Eratosth. Cat. 14; 23; Hyg. Astr. 2, 21; Exc. Tzetz. de Pleiadibus p. 551 (Martín); Sch. Hom II. 18, 486; Sch. Arat. 172.

23 Hyg. Astr. 2, 21; Sch. Arat. 174; Serv. Aen. 1, 744.

24 Pherecyd. F. 90b (FGrH).

25 Sch. Hom. Il. 18, 486; Sch. Arat. 254 (p. 201 Martin).

26 Manil. 4, 140-149, 380; 518-524.

27 Cf. pour les karpoi "fruits de la vigne": Hom. Il. 3, 246; 18, 561-568; Hdt. 1, 212.

28 Sch. Hes. Op. 571-577 (Pertusi).

29. Cf. les jours de mai indìqués par les auteurs: 5 (Gem. p. 107 Aujac); 10 (Colum. Rust. 11, 2, 40); 11 (West ad Hes. Op. 383 ss.); 13 (Excerpt. Tzetz de Pleiadibus p. 548 Martin); 14 (Eudox. F. 254 p. 204 Martin); H. Gundel, in RE s.v. Pleiaden 2511-2514; chez les Latins la datation varie entre le 22 avril et le 11 mai, cf. A. Le Boeuffle, Le nom Latin d'astres et de constellations, Paris 1977. p. 272.

30 Sch. Hes. Op. 383-387 (p. 130 Pertusi); 385a; 571-577; Sch. Arat. 254 (p. 204 Martin).

31 La floraison de son début à la chute des tleurs, dure 10-20 jours dans les vignobles, selon l'espèce des plantes (cf. B. Pastena, Trattato di viticoltura italiana, Bologna 1981, p. 199) et sa durée est fonction des conditions climatiques. Pour évaluer à peu près la durée de la floraison dans les vignobles thebains, iT peut être utile de savoîr que, dans là région de Palermo c'est ả dire environ à la même latitude, de nos jours, les vignes les plus précoces commencent à fleurir début mai (2-13) et, exceptionnellement, fin avril; les autres vignes vers le 10-21 mai et les vignes tardives le 14-26 mai; et que les grappes commencent à se former fin mai/début juin ou au plus tard vers la mi-juin, (cf. B. Pastena, loc. cit. p. 199;217;219; 957 s.).

32 Thphr. CP 3, 16. Pli. NH. 17, 190 Pampinatio verna in confesso est ab idibus Maiis, (15V) intra dies $X$, utique antequam florere incipiat. Colum. Rust. $11,2,44$. Ab idibus usque in calendas Iunias (15 V-1VI) veteranam vineam prius quam florere incipiat iterum fodere oportet eandem et ceteras et omnes vineas identidem pampinare,; Gp. 3, 5, 7; Colum. Rust. 11, 2, 37-38; Pallad. $6,2,1$. 

est eligendum sed et postea licet eandem repetere. Medium igitur eorum dierum spatium, quo ' acini formantur, vinearum nobis aditum negat quippe florentem fructum movere non expedit.

34 Colum. Rust. 4, 5; Arb. 12, 2.

35 Gp. 3, 4, 5; 5, 4; cf. B. Pastena, loc. cit., p. 923.

36 Thph. $C P .3,16,1-2$ (cit.). Gp. 3, 5, 7. Une inscription de lîle d'Amorgos (IG XII, 7, 62 vv. 810) exhortait de piocher les vignes avant les vingt derniers jours du mois du Taureau. Pli. NH. $17,188-189$ sat is esse ter anno confodi ab aequinoctio verno ad Vergiliarum exortum et canis ortu et nigrescente acino quidam ita determinant:...iterum ab idibus Aprilibus antequam concipiat hoc est in VI idus Maias dein prius quam florere incipiat et cum defloruerit et variante se uva.; Colum. Rust. 4, 28, 2 Nec infitior plerosque ante me rusticarum rerum magistros tribus fossuris contentos fuisse; ex quibus Graecinus qui sic refert: potest videri sat is et variante se uva.; Colum. Rust. 4, 28, 2 Nec infitior plerosque ante me rusticarum rerum magistros tribus fossuris contentos fuisse; ex quibus Graecinus qui sic refert:potest videri satis esse constitutam vineam ter fodere. Celsus quoque et Atticus consentiunt tres esse motus in vite...unum quo germinet; alterum quo floreat, tertium quo maturescat. Hos ergo motus censent fossionibus concitari.

37 Clem. Al. Protr. 2, 22 P.

38 Paus. $9,17,6 ; 10,32,10 \mathrm{~s}$.

39 Hom. Od. 11, 260 ss.; Apollod. 3, 10, 1; A.R. 1, 735 s.; 4, 1089s. Sch. ad. loc.

40 E. Antiop. F. 31 (Kambitsis); Ov. Met. 6, 110 ss.: Nonn. D. 7, 123; 16, 242; 31, 217; Sch. Stat. Theb. 9, 423; Myth. Vat. 1, 204.

41 Myth. Vat. 2, 74; Sch. Stat. Theb. 7, 189.

42 Paus. 1, 38, 8 ss.; cf. Hyg. F. 7; Myth. Vat. 1, 94; 2, 74.

43 Apollod. 3, 5, 5; Prop. 3, 15, 11; Hyg. F. 8; sur le Citheron elle aurait rencontré Dirké venue y célébrer les rites de Dionysos; cf. W. Luppe, Das Neue Euripides Fragment P. Oxy 3317, ZPE XLII, 1981, p. 27-30.

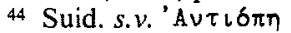

45 Sch. E. Ph. 102.

46 E. Antiop. F. 48 (Kambitsis) vv. 1-16.

47 E. Antiop. (loc. cit.) vv. 67-79; 86-103. cf. note 18.

48 E. Antiop. (loc. cit.) vv. 80-85; Amphion et Zéthos auraient jeté eux-mêmes le cadavre dans la source (Apollod. 3, 5, 5) que l'on disait être jaillie du corps (Sch. Stat. Ach. 1, 12) ou du sang de Dirké (Sch. Stat. Theb. 3, 205) à l'endroit oủ le taureau l'aurait transportée (Sch. E. Ph. 102). On disait aussi que les eaux de Dirké devenaient rouges pour annoncer un danger imminent aux Thébains (D. S. 17, 10, 4; Ael. VH. 12, 57; Stat. Theb. 4, 374 s.).

49 E. Pho. 101 s.; 825-827.

50 E. $B a .519-525$.

s1 S. Ant. 1115-1151; E. Ion. 1125 s.; Paus. 10, 64; 32, 7.

52 À propos d'Antiope prêtresse du temple du Soleil et Bacchante cf. Cephalio F. 5 FGr H; Georg. Cedr. Hist. Comp. 24 C; Weniger, dans Roscher, s.v. Phokos, 2412, voit une relation entre le menadisme d'Antiope (Paus. loc. cit.) et son arrivée à Tithorée.

53 Paus. 10, 1, 1; 2, 29, 3; St. Byz. s.v. $\Phi \omega$ \% lg

54 L'emplacement de la tombe des fils d'Antiope près des portes Proitides, coïnciderait avec la ressamblance entre leur mère et les filles de Proitos, lesquelles furent guéries d'une folie dionysiaque, et rendirent une source "ennemie de la vigne" et épousèrent Melampous, la guérisseur, et son frère; cf. Apollod. 2, 2, 2; Ov. Met. 15, 322, ss.; St. Byz. s.v. 'A $\zeta \alpha$ v i $\alpha$ MK. Massenzio, Xenia dionisiaca, SMSR XL, 1969, p. 97-104; P. Scarpi, Melampous e i "miracoli" di Dionysos, Perennitas. Studi in onore di A. Brelich, Roma 1980, p. 435-440.

55 Thyios, à Thèbes (IG VII 2423) comme dans le calendrier béotien, était le conquième mois de l'année et commençait après le solstice d'hiver; cf. P. Roesch, Études Béotiennes, Paris 1982, p. $46 ; 54$.

s6 $\grave{A}$ propos des Thyiai "femmes folles du dieu" sur le Parnasse, cf. Paus. 10, 6, 4; $32,7$.

57 Cf. l'identification de la constellation des Didymoi avec les Dioscures dans Eratosth. Cat. 10; Hyg. Astr. 2, 22; et en particulier avec Amphion et Zéthos dans Sch. Germ. BP68, 6; Anth. Lat. 626, 2 (Riese). 


\section{Summary}

Pausanias reports that every year when the Sun was passing through the constellation Taurus, Tithoreans planned to steal earth from the tomb of Amphion and Zethos in Thebes of Boeotia.

Bringing this earth in their city and pouring it over Antiope's tomb they would have favoured the fertility of their fields. In order to preserve the fruits of their country and following the oracle of Bakis, Thebans kept watch over the tomb of Amphion and Zethos.

An analysis of Pausanias' text suggests that a quarrel divided the two cities, every part claiming the right to have in its land the tomb of Antiope's sons. The involvement of the mythical figures and the peculiar phase in the vineyards' life during that period of the year (April-May) both suggest that Thebans tried to protect the growth of the fruits of Dionysos.

\section{Résumé}

Chaques année, quand le Soleil se trouvait dans la constellation du Taureau, les Phocidiens de Tithorée essayaient d'offrir des libations sur le tumulus thébain d'Amphion et de Zéthos, et d'en dérober de la terre afin de la répandre autour de la tombe d'Antiope qui se trouvait chez eux, et ceci pour appeler la fertilité sur leurs champs.

De leur côté, les habitants de Thèbes, suivant en cela l'oracle de Bakis, s'efforçaient d'empêcher l'action des Phocidiens pour préserver les fruits de leurs propres champs.

Le passage de Pausanias laisse envisager l'existence d'une controverse entre les deux cités pour la possession du tumulus, les Thébains repoussant chaque année les prétentions des Tithoréens qui auraient pu bouleverser l'ordre établi par les dieux.

Les références aux personnages mythiques; le mois en question ainsi que les travaux des vignerons à ce moment de l'année, tout porte à croire que les fruits dont on voulait protéger la récolte étaient les fruits de Dionysos. 